\title{
WestVirginiaUniversity
}

THE RESEARCH REPOSITORY @ WVU

Graduate Theses, Dissertations, and Problem Reports

1999

\section{Automatic selection of optimal window size and shape for texture analysis}

Joseph Edmund Glotfelty

West Virginia University

Follow this and additional works at: https://researchrepository.wvu.edu/etd

\section{Recommended Citation}

Glotfelty, Joseph Edmund, "Automatic selection of optimal window size and shape for texture analysis" (1999). Graduate Theses, Dissertations, and Problem Reports. 849.

https://researchrepository.wvu.edu/etd/849

This Thesis is protected by copyright and/or related rights. It has been brought to you by the The Research Repository @ WVU with permission from the rights-holder(s). You are free to use this Thesis in any way that is permitted by the copyright and related rights legislation that applies to your use. For other uses you must obtain permission from the rights-holder(s) directly, unless additional rights are indicated by a Creative Commons license in the record and/ or on the work itself. This Thesis has been accepted for inclusion in WVU Graduate Theses, Dissertations, and Problem Reports collection by an authorized administrator of The Research Repository @ WVU. For more information, please contact researchrepository@mail.wvu.edu. 


\title{
Automatic Selection of Optimal \\ Window Size and Shape \\ for Texture Analysis
}

Joseph Edmund Glotfelty

Thesis submitted to the College of Arts and Sciences

at West Virginia University

in partial fulfillment of the requirements

for the degree of

\author{
Master of Arts \\ In \\ Geography
}

Committee Members:

Dr. Joseph Gardner

Dr. Robert Hanham

Dr. Timothy Warner, Chair

Department of Geology and Geography

Morgantown, West Virginia

1999

Keywords: Texture, Windows, Edge Effect

Copyright 1999 Joseph E. Glotfelty 


\section{$\underline{\text { ABSTRACT }}$ \\ Automatic Selection of Optimal Window Size and Shape for Texture Analysis}

Joseph E. Glotfelty

This thesis is a theoretical and empirical examination of the relationship between texture and scale, and its effects on image classification. This study involved creating a model that automatically selected windows of optimal size according to the location of a pixel within a land cover region and the texture of the surrounding pixels. Large windows were used to get a representative sample of within-class variability in the interior of these regions. Smaller windows were used near the boundaries of land cover regions in order to reduce "edge effect" errors due to between-class variability. This program was tested using a Maximum Likelihood classification scheme against spectral data and texture from fixed-size windows to determine if there were any improvements in classification accuracy. Three different types and scales of data, including SPOT, SIR-C, and ADAR, were used to test the robustness of this program.

The results from this research indicate that the addition of texture can improve classification accuracy, especially in land cover regions with high local variability among the pixels. The $21 \times 21$ texture image achieved a Kappa Index of Agreement (KIA) of 0.97 for the highly textured Sunlit Forest (leaf off) class in the ADAR data, compared to 0.92 using the spectral data alone. However, texture windows of fixed-size created some errors due to "between-class" texture. This was most evident in the SPOT interior test data where the $21 \times 21$ texture window achieved a KIA of 0.70 , compared to 0.92 for the spectral data. In many cases, images that incorporated the Optimal Size Window 
Program were superior in accuracy to all of the other images. In the radar data, the image created from the Optimal Size Window Program improved the overall KIA from 0.51 in the spectral data, to 0.71 . 


\section{$\underline{\text { ACKNOWLEDGEMENTS }}$}

I would like to thank all of my friends in the Geology and Geography Department for all of the helpful support and encouragement that they have given me. I also would like to thank Dr. Joseph Gardner, Dr. Robert Hanham, and Dr. Timothy Warner for taking the time to review my work and serve on my committee. Without the help of my friends and faculty in the Geology and Geography Department, this thesis would not have been possible. 


\section{TABLE OF CONTENTS}

ABSTRACT ….............................................................................. ii

ACKNOWLEDGEMENTS ........................................................ iv

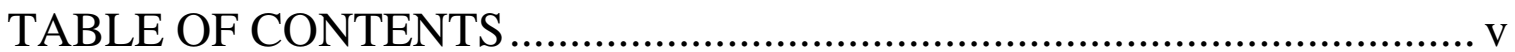

LIST OF FIGURES ................................................................... vi

LIST OF TABLES ................................................................... vii

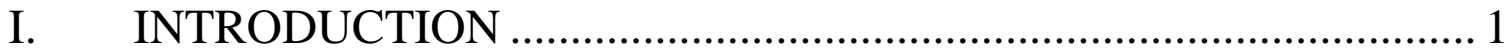

II. THE SPATIAL PROPERTIES OF IMAGES ................................. 4

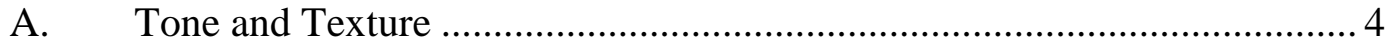

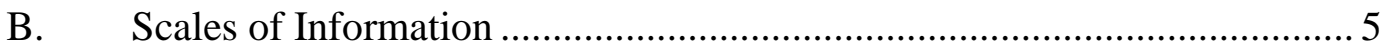

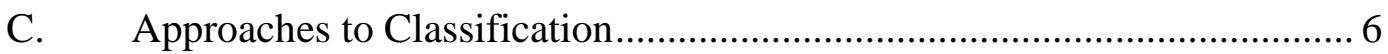

D. Approaches based on Systematic Patterns …......................................... 8

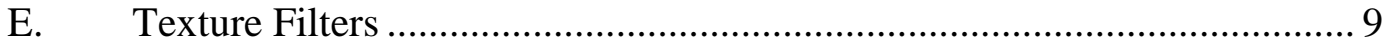

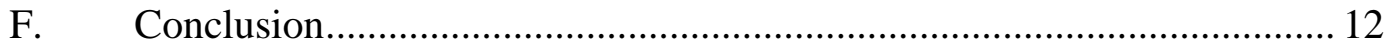

III. RESEARCH OBJECTIVES ................................................ 13

IV. METHODS ...................................................................... 15

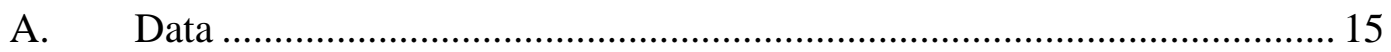

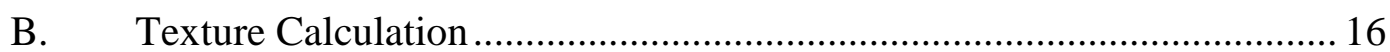

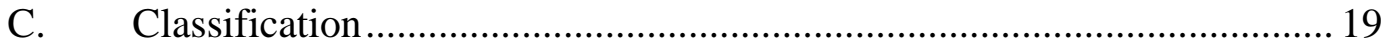

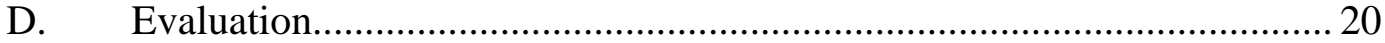

V. A RULE-BASED APPROACH TO MEASURING TEXTURE...... 23

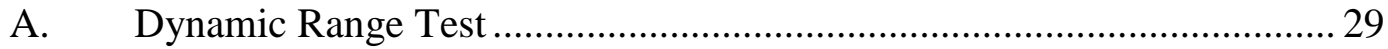

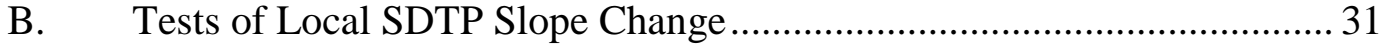

C. Adaptive Minimum Window Program.................................................... 34

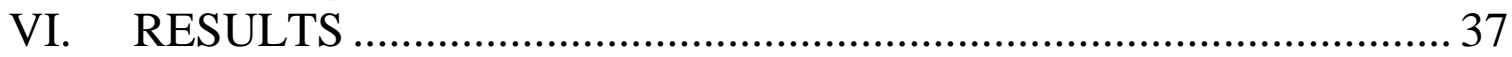

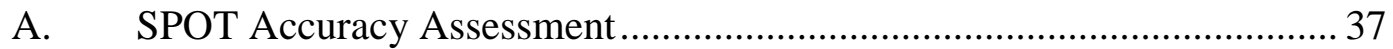

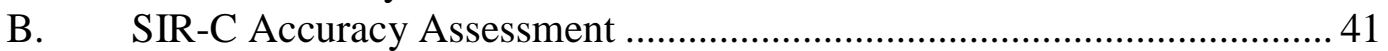

C. ADAR Accuracy Assessment ........................................................... 45

VII. CONCLUSIONS ..................................................................... 49

VIII. RECOMMENDATIONS FOR FURTHER RESEARCH................ 52

IX. REFERENCES ................................................................ 55 


\section{LIST OF FIGURES}

Figure 1: SPOT Image of Cheat Lake ...................................................................... 18

Figure 2: Land Cover Boundary on SDTP Curve ................................................... 24

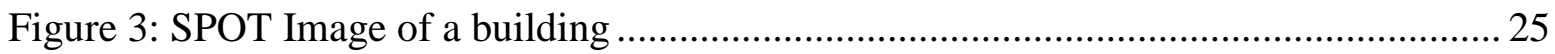

Figure 4: ADAR False Color Composite of Shaded Forest .......................................... 26

Figure 5: SPOT Image of a residential area ....................................................... 27

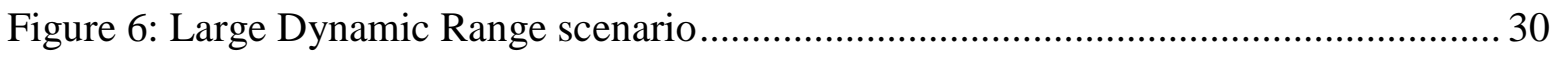

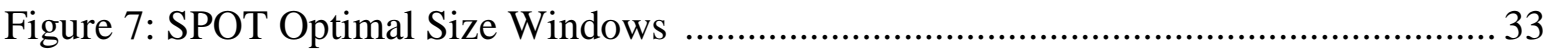




\section{$\underline{\text { LIST OF TABLES }}$}

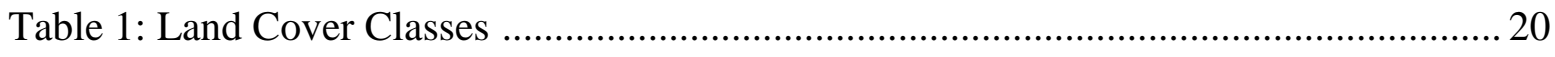

Table 2: Rules for the Optimal Size Window Model................................................... 28

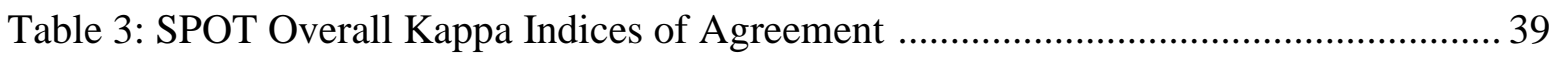

Table 4: SIR-C Overall Kappa Indices of Agreement ............................................... 43 


\section{CHAPTER ONE INTRODUCTION}

Medium resolution sensors such as the LANDSAT Multi-Spectral Scanner (MSS) and Thematic Mapper (TM) have been shown to be very effective for digital classification and mapping of land cover. However, as sensor technology has become more sophisticated, the spatial resolution of more recent sensors has increased to the point where a new, finer scale of objects can be resolved, especially in built-up and urban areas. Consequently, these classes display a greater variability because, as the pixel size approaches the size of the individual objects on the ground, variability between neighboring pixels naturally increases (Woodcock and Strahler, 1987). In these circumstances, high resolution data actually leads to lower classification accuracy (Latty et al., 1985). Furthermore, the highest resolution satellite-borne sensors usually have limited spectral resolution from only a single band (Kramer, 1996), making it difficult to use such data in digital multispectral classification. Consequently, researchers are showing renewed interest in the spatial properties of images. This interest is also driven by the excessive high frequency noise in many aspatial classifiers. Some studies have successfully used post-classification, rule-based approaches to merge segments into larger regions by using properties such as area, perimeter, and size (Johnnson, 1994). However, these approaches are very site-specific and are not robust enough to use in different geographic locations or with different scales and types of data. Finally, by relying solely on the spectral characteristics of images, valuable spatial information is being discarded. 
Most of the limitations of using spectral classifiers are due to information being extracted at a single scale of measurement determined by the pixel size. By using the spatial properties and relationships that exist between pixels, information can potentially be extracted at a greater variety of scales. However, many current approaches have focused mainly on algorithm development, and less so on the scale at which spatial information will be extracted. Yet, a number of studies have found that the scale is crucial. A large window is necessary to capture a sample that represents the within-class variability of the population. However, large windows can overlap onto neighboring land cover regions, leading to significant between-class errors. An important area of research is the search for a method that accurately measures texture at a variety of scales.

This thesis examined the value and feasibility of collecting texture information from a window that is optimally-sized to maximize the measurement of within-class variation, and reduce error due to between-class variation. It consists of two parts. First, a model was developed that consists of a set of rules for estimating the optimal scale of texture measurement based on the location of the central pixel relative to the inferred position of surrounding land cover boundaries. The texture images derived from this model were then evaluated by running classifications on the data and comparing them to classifications of images using only spectral information and images using texture information derived from fixed-size windows. The evaluation involved both qualitative and quantitative assessments to determine the benefits of using this model over a variety of types and scales of data.

Chapter 2 lays out a theoretical framework by providing a discussion of tone and texture, and how these spatial properties are being examined in the current research. The 
Research Objectives for this thesis are listed in Chapter 3. This is followed in $\underline{\text { Chapter } 4}$ by a description of the Methods that were used in this research. The development of the rules for the Optimal Window Model and the Adaptive Window is discussed in Chapter 5. The Results of this research are then analyzed using both qualitative and quantitative assessments of classification accuracy in Chapter 6. Finally, Chapter 7 discusses the contribution of knowledge of these findings in the broader context of ongoing research in Remote Sensing 


\section{$\underline{\text { CHAPTER TWO }}$}

\section{THE SPATIAL PROPERTIES OF IMAGES}

\section{TONE AND TEXTURE}

Two common properties that are sometimes used to characterize spatial relationships are tone and texture. Understanding of these two properties is crucial for their effective application. Tone can be conceptualized as a single, unifying value or quality that results from the combination of the unique elements and attributes that constitute an entity or recognizable object. Tonal qualities dominate an entity when the scale of observation is too coarse to allow the many elements that comprise it to be resolvable individually. Thus, tone is the dominant quality at a given scale and becomes the unit of measure when finer scale elements are unresolvable. Therefore, the entire population, rather than individual elements, is the unit of interest when measuring tonal properties. Texture can be thought of as orthogonal to tone. Rather than acting as a measure of the similarity or unification of elements as with tone, texture is a measure of differences in these elements. The arrangement and attributes of these individual elements form a structure. Thus, when measuring texture, the unit of interest is in these individual elements and their relationships with one another. In spatial terms, texture is generally thought of as an array of tonal values across an $\mathrm{x} / \mathrm{y}$ planar coordinate system that differ from one another, yet form a pattern that can sometimes allow a larger entity to be recognized. 
Tone and texture are not mutually exclusive properties but rather form a continuum whereby, as resolution is changed for a given area, one is gradually replaced by the other as the dominant property that is apparent at a particular scale. If scale is decreased and spatial resolution becomes lower, the unique attributes of the elements (texture properties) gradually become eroded, and these attributes become combined or generalized into a larger entity that is better characterized by its tonal properties. Thus, tone is actually composed of a finer-scale texture that gradually becomes apparent as resolution is increased. Also, texture is actually composed of individual objects, each with its own specific tonal value.

\section{SCALES OF INFORMATION}

In order to understand how the tonal and texture properties arise in an image, it is necessary to differentiate between the ground scene, and its abstraction represented by the image. Elements in the image become apparent at different spatial scales of imaging. These elements are made up of spatially dependent components with similar colors and textures. The Nested Hierarchical Scene Model has been proposed as a way of describing these elements at various scales (Collins et al., 1995). Each level of the original scene decomposes to a new level as scale is changed. An example might be a forest at one level, which decomposes to an individual tree at the next level of detail. The tree then decomposes to clusters of leaves, and then single leaves. This decomposition is infinite. However, the digital image representation of a scene is limited to the spatial resolution of the pixels. There are three possible relationships that exist between pixel resolution and the size of an object on the ground (Woodcock and Strahler, 1987). The first is a condition known as H-Resolution. In this case, the size of the pixel is much smaller than the object of interest. Thus, an object is made up of a number of pixels. The individual 
pixels generally exhibit high spatial autocorrelation between one another. In the second case, the pixel size is at or near the size of the object. The highest local variance in pixel neighborhoods occurs when pixels are fifty to seventy-five percent as large as the object of interest. Finally, when pixel size is larger than an object, a condition known as LResolution takes place. Under this condition, the large size of the pixels "absorbs" the local variance within the scene by integrating the different reflectances of all of the objects in the Instantaneous Field of View (IFOV) into a single measurement value. Thus, the H-and L-Resolution classes are the most homogenous, while the pixels that are nearly the same size as the objects exhibit the most heterogeneity or texture (Woodcock and Strahler, 1987). In a typical LANDSAT TM scene, a forest stand might be considered to be in H-Resolution, the individual trees in L-Resolution, and buildings would be nearly the same size as the pixel size.

A ground scene may have a number of land cover classes or objects of interest at a particular scale. In the context of the Nested Hierarchical Scene Model of Collins et al. (1995), the texture of each of these classes is composed of the smaller elements that make up that particular class. These elements at one scale may become the elements of interest at a finer scale. Thus, because of this hierarchical relationship that exists between a land cover class and the elements that comprise it, texture may exist at several different scales within one land cover class. This is an important fact to consider in approaches that use moving window filters to create texture images.

\section{APPROACHES TO CLASSIFICATION}

Due to the low spatial resolution of early sensors, approaches to image

classification based on tonal values have been used extensively over the past three decades. The large pixel size in relation to objects on the ground had the effect of 
creating large, relatively homogenous regions of pixels with similar tonal values. This led to the emphasis on methods that used the average grey-level values of classes for classification of land cover classes.

As new generations of sensors that have finer spatial resolution have become available, the pixels have become smaller relative to the ground objects, resulting in greater variation among pixel grey-level values, especially in urban and built-up areas. This greater variation among the pixels has increased the importance of incorporating texture into image classification. As mentioned previously, texture is associated with individual elements and their spatial arrangement. This spatial arrangement of elements can be conceptualized as having three measurable components: distance, direction, and intensity. Patterns in one or all three of these components can serve as a source of information, and form the basis of measurable attributes. Depending on the interplay of objects at different scales, these spatial arrangements can have patterns that range on a continuum from entirely random to entirely systematic. Rarely are patterns entirely random or systematic. Rather, there is nearly always some degree of both of these qualities that exist within an entity. Patterns that are more random have a low degree of correlation among elements. At one scale patterns that are apparent may be dominated by randomness but, at a finer scale, spatial relationships may exist that are more systematic. The existence of these intrinsic systematic relationships can allow information to be extracted using methods that employ statistics. Patterns that are systematic consist of elements that are associated with one another. In some cases, contextual relationships that are directly observable may dominate an entity. An example of a systematic pattern would be a building and its associated shadow. Some methods 
using ancillary data to delineate co-occurrence can be very powerful tools for extracting spatial information from an image. However, these contextual relationships are usually scene specific. All methods that employ texture in image classification use a combination of both statistics and context to some degree. Yet, some of these methods are more useful for measuring systematic patterns while others are more useful for measuring random patterns.

\section{APPROACHES BASED ON SYSTEMATIC PATTERNS}

Texture methods that are based on systematic properties of texture are most effective on areas of an image that have land cover regions with elements that are nearly the same size as the pixels. These approaches generally rely upon the spectral similarity or co-occurrence of pixels at a given direction and/or distance.

Fourier Analysis is an approach to spatial analysis that identifies periodic relationships in an image through the use of a constructed frequency spectrum. Riou and Seyler (1997), used this method to map lineament patterns and forest canopy structures in the tropics. A major drawback to Fourier Analysis is that it relies heavily upon some periodicity or repeatable pattern to be present. In many cases, texture within an image can be chaotic or random.

In another approach, Hay et al., (1996) used the triangulated primitive neighborhood method to classify stands of trees by determining the size and spatial arrangement of individual trees. First, the highest DN values in local neighborhoods were identified as tree crowns. The crowns were then connected by straight lines to form triangles between neighboring crowns. These triangular primitives were then used to determine the densities of stands. 
Several studies have used the range or shape of the semivariogram to infer spatial attributes from an image (Woodcock et al., 1988a; 1988b). In one study, illegal waste disposal sites were separated from spectrally-similar landfills and quarries by comparing semivariograms (Brivio et al., 1993). Semivariograms have also been used to separate different-aged forest stands by examining canopy size and structure. For example, the semivariance along a linear transect of pixels allowed researchers to differentiate old growth stands from the more regular pattern of young stands (Cohen et al., 1990).

One of the earliest and most commonly used methods for systematic spatial analysis is the Grey Level Co-occurrence Matrix (GLCM) (Haralick and Shanmugan, 1974). An example of the application of the GLCM is Barber and LeDrew's (1991) study of texture discrimination between different ages of sea ice in Synthetic Aperture Radar (SAR) data. The GLCM is especially useful for analyzing SAR data since the variation in radiometric response throughout the image eliminates the possibility of using average tonal response in the classification. In yet another approach, Wang and $\mathrm{He}$ (1990) used an approach that generates for each pixel a Texture Unit, which is a measure of the local texture in eight different directions from a central pixel. The ordering of these differences around a given pixel forms a unique identifier for the local texture and, becomes the Texture Unit value.

\section{Texture Filters}

A major limitation to the use of some of the approaches mentioned above is that they rely, to some degree, on systematic patterns to extract spatial information. However, in many cases, especially when objects are in L-Resolution or are similar in size to the pixel IFOV, random variation is a predominant property between pixels in many land cover regions. In this circumstance most pixels are "mixed" pixels, with DN values that 
are not really representative of any given object, but rather, are the result of the combining of many objects' reflectances. Therefore, an approach that attempts to measure these individual objects may not be appropriate under these conditions.

Moving texture filters transform the local variability within the window into a new information channel that potentially can be incorporated in conventional spectral classification. This is accomplished by taking a measure of dispersion, such as the range (Briggs and Nellis, 1991) or standard deviation of a moving window, and placing this value in the central pixel (Jensen, 1979; Agbu and Nizeyimana, 1991). This creates a new image, with each pixel representing the local standard deviation value. The complex nature of texture within an image has led to much research concerning how best to capture the local variability between the pixels. Marceau (1990) found that $90 \%$ of variability was accounted for by the size of the window, whereas types of algorithms used to calculate texture only accounted for $7 \%$. Yet, until recently, most studies have focused more on algorithm development and, very little research has focused on the appropriate size of the windows used to derive texture (Hodgson, 1998). The size of the window is so important because large window arrays are needed to increase the number of samples, to lower the variability of the texture values within each class and thus increase the separability between classes. However, the larger the window, the more it will overlap two or more land cover regions and lead to significant classification errors near the edges of these regions (Ferro, 1998). This has been termed the "edge effect". To avoid this phenomenon, most researchers have used smaller filters, thus reducing the overlap of the window between two land cover regions (Hsu, 1978; Dutra and Mascarenhas, 1984; Gong, et al., 1992). Dutra and Mascarenhas (1984) found that the 
use of smaller filters was preferable to preserve "microtextures" within an image.

Adaptive filters have been used to counteract the edge effect problem. One

implementation of an adaptive filter is accomplished by first running a moving window filter across an image to create a texture image. A second moving window filter is then run over the new texture image and, the lowest value in the window is placed into the central pixel (Ryherd and Woodcock, 1996). However, this adaptive filter can lead to a blocky texture across the image because unusually low values tend to dominate in the second part of the filtering.

Another problem involving the selection of an appropriate window size has been caused by elements with diverse shapes within an image that can exist at a very large number of scales. Several studies have suggested that no single window size is optimal across an entire image (Chavez and Bauer, 1982; Ahearn, 1988; Franklin and McDermid, 1996). Schunck (1987) used multiple window sizes to derive the texture of forests in Kluane National Park, Yukon Territory, Canada. Rather than relying upon "geometric windows" of fixed in size and shape, Merchant (1984) proposed the idea of using a "geographic window" that changes size and shape to conform to the local landscape characteristics. This approach, however, required image classification be carried out prior to the texture analysis. Dillworth et al., (1994) used this geographic window concept to measure landscape characteristics such as patch size, diversity and interspersion. In many cases, dramatic changes in variation are prevalent at the boundaries between two land cover regions. Palmer and Petrou (1997) used these abrupt changes in variation to determine the location of edges between land cover types. In this particular case, the concept of a free angle between pixels of high variance was used to 
delineate boundaries between regions of high variability and low variability. These free angles are largest when facing a homogenous land cover type and, smallest when facing a highly textured land cover type. Another study, by Hay et al., (1997) used the inflection point of a spatially-dependent profile curve of a number of different window sizes to determine the optimal size window to use for upscaling the data. Other studies have used the range of semivariograms to customize the appropriate window size for classification (Franklin and McDermid, 1993). Hodgson (1991) used multiple windows of a variety of sizes and shapes to characterize the homogeneity of a landscape.

\section{Conclusion}

Texture filters have been extensively used in a variety of applications. They can be a simple method for measuring random spatial patterns in images. However, conflicting requirements due to scale of objects within an image have limited their usefulness. Large windows are needed to get a representative sample of pixels. Yet, these large windows can lead to substantial errors due to edge effect. Smaller windows may not take large enough samples to fully characterize the texture of a particular land cover region. Windows of multiple sizes have been used for a variety of applications, and may offer a potential solution to the conflicting requirements of scale. 


\section{CHAPTER THREE}

\section{RESEARCH OBJECTIVES}

Two major conflicting requirements arise when using a moving window filter to convert variation between the pixels into a texture measure that gives the maximum separability between the classes and the minimum within-class variability (Ferro, 1998). Large windows are required to ensure a large number of samples within each window, and to smooth local neighborhood variation in favor of larger scale trends in the data. However, by using large filters, the between-class texture tends to overwhelm withinclass texture (Ferro, 1998). Furthermore, the analyst may miss finer scale "microtextures" that may exist in the data (Dutra and Mascarenhas, 1984).

The primary objective of this research was to investigate adaptive texture measures, and to determine if such methods can be used to improve classification accuracy by overcoming the conflicting requirements of the fixed window. Filters that are adaptive in both size and shape were studied. Three main hypotheses were addressed:

1. Does a filter that selects the "optimal" window size for calculating the texture of each pixel offer improved accuracy over a fixed-size window?

2. Does the inclusion of optimal shape algorithms improve the accuracy of this optimal size filter?

3. Can this optimal window filter be designed to be robust enough to be useful across many types and scales of data?

Three images presenting contrasting scales and data types were used to investigate the research questions. Those images, and texture derived from them, were 
classified and the accuracy of the classification used to evaluate the usefulness of the texture channels. The classification included:

1. Spectral data only.

2. Spectral data and fixed window size texture.

3. Spectral data and texture from optimal-sized windows.

4. Spectral data and texture from optimal-sized and adaptive windows. 


\section{CHAPTER FOUR METHODS}

The overall research objective was to investigate adaptive texture measures, and to determine if such methods could be used to improve classification accuracy by overcoming the conflicting requirements of the fixed windows. Specifically, the aim was to create a single texture channel from the optimal size and adaptive windows. This new texture channel was then combined with other bands in a supervised spectral classification scheme that used a Maximum Likelihood decision rule to separate the spectral classes into new informational classes (Swain and Davis, 1978; Richards, 1996). The classified images were then evaluated, both qualitatively and quantitatively, to determine if the incorporation of texture changed the accuracy of the classification. Accuracy was tested across entire land cover polygons, including the edge areas, which are particularly prone to errors when texture is used in classification (Ferro, 1998).

\section{Data}

Three different types and scales of data were used in order to provide a thorough evaluation of the robustness of the Optimal Window Program. This data was acquired in digital format, and only portions of entire scenes were used. All three data types were from the Morgantown area, but did not did precisely correspond geographically. Also, the acquisition dates and times did not correspond. Therefore, the three data types had large differences in spatial, spectral, and temporal characteristics. These differences were intentional so that the robustness of this approach, rather than the differences between the data, could be examined. First, a Systeme Pour l'Observation de la Terre (SPOT) HRV image of the Morgantown, West Virginia area with 20 meter pixel resolution was 
obtained. This image was acquired on 16 August 1987 with full leaf cover, and consisted of data collected in three bands: $0.50 \mu \mathrm{m}$ to $0.59 \mu \mathrm{m}, 0.61 \mu \mathrm{m}$ to $0.68 \mu \mathrm{m}$, and $0.79 \mu \mathrm{m}$ to $0.89 \mu \mathrm{m}$.

Secondly, a Shuttle Imaging Radar (SIR-C) image of the Morgantown, WV area was obtained. This Synthetic Aperture Radar (SAR) operated in the L-Band (23.5 centimeter wavelength, $1.249 \mathrm{GHz}$ frequency) of the microwave portion of the electromagnetic spectrum. Two bands were acquired: vertical transmit/vertical receive (VV) and vertical transmit/horizontal receive $(\mathrm{VH})$. The image was acquired on September 9, 1994 at 18:12 GMT with full leaf cover.

Thirdly, a mosaiced Airborne Data Acquisition and Registration (ADAR) image of the Morgantown, WV area was obtained. This image has one meter resolution and spectral information from four bands: $0.45 \mu \mathrm{m}$ to $0.54 \mu \mathrm{m}, 0.52 \mu \mathrm{m}$ to $0.60 \mu \mathrm{m}, 0.61 \mu \mathrm{m}$ to $0.69 \mu \mathrm{m}$, and $0.78 \mu \mathrm{m}$ to $1.0 \mu \mathrm{m}$. This orthomosaic was acquired over the period from March 24, 1997 to April 2, 1997. There was no deciduous leaf cover at the time of acquisition.

\section{$\underline{\text { Texture Calculation }}$}

The texture images used in this research were created from each of the three original images. To simplify computation, the multiple bands in each of these three images were reduced to single bands using the first principle component from a Principle Components Analysis (PCA). The first principle component, corresponding to brightness and illumination, was used because it was responsible for much of the image texture. Spectral differences contained in the second principle component were found to be less important. Ten sizes of windows $(3 \times 3,5 \times 5,7 \times 7,9 \times 9,11 \times 11,13 \times 13,15 \times 15$, $17 \times 17,19 \times 19$, and 21x21) were used in the Imagine Spatial Modeler to create ten new 
images of texture values. The standard deviation from the mean of all pixels within each window was used as the texture measure. By moving this window across an entire image, new images with tonal contrasts based on the local texture of the original image were created.

The ten texture images were combined using the STACKLAYERS command in the SPATIAL MODELER to create a model to select optimally-sized windows. The SPECTRAL PROFILE TOOL was used to observe the scale-dependent pattern of texture by graphing the texture values through the layer stack for a particular pixel. The standard deviation was plotted along the ordinate, and the window size (from smallest to largest) was plotted along the abscissa. As mentioned previously, one of the major drawbacks associated with using large moving windows is that they can overlap two land cover classes and produce significant errors. The Scale Dependent Texture Profile (SDTP) curve allowed boundaries between land cover regions to be detected through the abrupt changes in the slope of the curve.

The curves for many of the pixels throughout the image had a characteristic asymptotic shape, which was mainly due to the exponential relationship increase in the number of pixels in the window as the window size increases. For example, an edge of a land cover class may cover a significant proportion of pixels in a small window, but may have less of an impact on the standard deviation of a larger window. This resulted in the changes in slope due to these edges typically being more severe or abrupt in the smaller windows than in the larger ones. For example, a boundary might take up three of the nine pixels $(33 \%)$ in a $3 \times 3$ window. The same boundary on the outer edge of a $21 \times 21$ window may account for only 21 of the 441 pixels (21\%). Thus, a boundary that occurs 
on the SDTP curve between two small windows such as $3 \times 3$ and $5 \times 5$, would have a much steeper slope than one that occurs between larger windows such as $19 x 19$ and 21x21. This is illustrated in Figure 1.
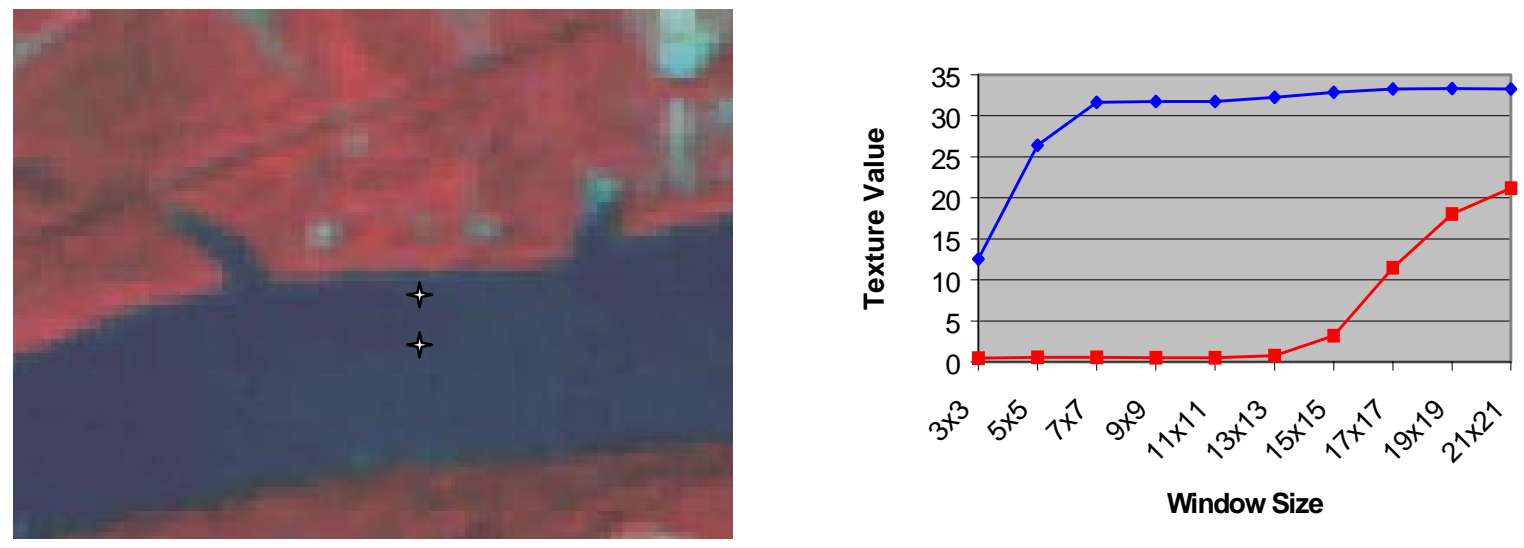

Figure 1: Left: Standard False Color SPOT image of shoreline of Cheat Lake, WV. Right: Graph of the SDTP curves of two different pixels in SPOT image on right. Note the asymptotic relationship between the windows of different sizes.

Figure 1 shows a portion of a SPOT image and a graph of the SDTP curves of two pixels from the image. The first pixel, represented by the blue curve, is located two pixels $(40 \mathrm{~m})$ from the shoreline of a lake. The three pixels in the top row of the $3 \times 3$ window for this pixel contain mixed pixels where the shoreline meets the lake. This accounts for the relatively high texture value of 12.5 for this window. The $5 \times 5$ window for this pixel has a much higher texture value of 26 , since the five pixels along the top row of the window contain forest pixels and the second row contains mixed pixels.

The second pixel is located eight pixels from the shoreline and is represented by the red SDTP curve. The top row of the $15 \times 15$ window contains the mixed pixels of the shoreline and has a texture value of 3 , while the top row of the $17 \times 17$ window contains 
forested pixels and its second row contains the mixed pixels, resulting in a texture value of 11. From this example, it can be seen that smaller windows containing an edge have much higher texture values than larger windows containing the same edge.

By identifying edges between land cover classes using the SDTP curve, it was possible to design a program that used a window that was optimally-sized. That is, it was as large as possible without overlapping the inferred position of another land cover class. Identification of boundaries was crucial for rule development associated with the Optimal Size Window Program. The abruptness of these boundaries was influenced by the boundary gradient between the two land cover regions and the asymptotic shape of the SDTP curve. The development of the Optimal Size Window Program and the Adaptive Minimum Texture Window Program is discussed in Chapter Five.

\section{Classification}

In order to provide an evaluation of the effectiveness of the texture measures, land cover classification was performed on the texture images. First, for all three types of data (SPOT, SIR-C, and ADAR), all of the multi-spectral and texture images were combined into a single image using the Imagine STACKLAYERS command. For example, the ADAR layer stack contained the following nine bands: blue, green, red, infrared, $3 \times 3$ window texture, $11 \times 11$ window texture, $21 \times 21$ window texture, optimal window size texture, and optimal window size/adaptive minimum texture.

Training samples were then collected from each of the three combination images using the Imagine SIGNATURE EDITOR (Table 1). This involved selecting a group of pixels from each desired informational class. The following table lists the classes for each type of image. 
TABLE 1: Land Cover Classes used for each type of data.

\begin{tabular}{|l|l|l|}
\hline \multicolumn{1}{|c|}{ SPOT } & \multicolumn{1}{|c|}{ SIR-C } & \multicolumn{1}{c|}{ ADAR } \\
\hline WATER & SHADOWS/WATER & GRASS \\
FOREST & URBAN & BARREN \\
GRASS/CROPS & RESIDENTIAL & FOREST (SUNLIT) \\
SPARSE GRASS & FOREST & FOREST (SHADED) \\
BARREN/URBAN & GHADOW \\
RESIDENTIAL & & WATER \\
COAL/MINING & & ROOFTOP (DARK) \\
& & ROOFTOP (LIGHT) \\
& & PAVEMENT/CONCRETE \\
& & ASTROTURF \\
\hline
\end{tabular}

After the training samples were collected from each class, the images were classified using the Maximum Likelihood decision rule. For each data type (SPOT, SIR-C, and ADAR), there were six classified images, one for each of the following data combinations:

1. Spectral data only.

2. Spectral and 3x3 Fixed Size Window Texture.

3. Spectral and 11x11 Fixed Size Window Texture.

4. Spectral and 21x21 Fixed Size Window Texture.

5. Spectral and Optimal Size Window Texture.

6. Spectral and Optimal Size/Adaptive Window Texture.

\section{Evaluation}

The classified images were evaluated for accuracy using both qualitative and quantitative methods. The qualitative evaluation based on a visual estimate of the accuracy is useful because it potentially can provide insight into causes of errors. Aerial photographs, ground truthing, and local knowledge were used in this process.

Two different quantitative methods were used to evaluate the effect that the incorporation of texture had on the accuracy of the classification. The first method was to compare the class statistics for each cover type. If the standard deviation of the texture was reduced, then the incorporation of texture could potentially have increased the separability of this class from the others. 
The second quantitative method that was used involved the construction of error matrices to determine the replicability of the accuracy of a particular classified image. This was achieved by selecting test samples of each class in a manner similar to the selection of the training data. However, rather than classifying the image on the basis of these samples, these test site polygons were compared to the classified image using the ERRMAT program in the IDRISI software package. This program created error matrices that allowed agreement and disagreement to be examined on a category by category basis between the classified images and known reference data (test data) that was collected from each land cover region (Jensen, 1996). Test data is represented by the columns, and the actual classified image data is represented by the rows. Agreement between the pixels in both data sets is represented in the major diagonal in the matrix. Errors of Omission can be found in the nondiagonal column elements, and are expressed as a percentage at the bottom of each column. These were pixels from a known test class that were omitted and misclassified as another class in the classified image. Errors of Commission can be found in the nondiagonal row elements, and are expressed as a percentage at the extreme right of each row. These were test pixels that were mistakenly included in a particular land cover class of the classified image. An overall measure of the classification performance is the Kappa Index of Agreement (KIA). This is obtained by dividing the total number of correctly classified pixels by the total number of test pixels (Lillesand and Kiefer, 1994).

The classified images were tested against three different types of test data. First, test pixels were selected from areas within ten pixels of the boundary between two classes. Ten pixels represents the length at which the largest windows would begin to 
overlap onto a neighboring land cover region. Next, test pixels were selected from the interior (greater than ten pixels from the edge) of land cover regions. Finally, test data were selected from both the edge and interiors of land cover regions. The primary goal of using these criteria for selecting test data was to assess effects that the optimal size and adaptive minimum programs had on reducing edge effect errors when compared to the fixed size windows. 


\section{CHAPTER FIVE}

\section{$\underline{\text { A RULE-BASED APPROACH TO MEASURING }}$ TEXTURE}

The primary goal of the Optimal Size Window Program was to use as large a window as possible without overlapping a class boundary. In order to accomplish this, it is crucial to differentiate between within-class variability and between-class variability. This model measures the changes in the slope of the SDTP curve to differentiate between these two types of textures. Since avoiding the measurement of between-class texture is of the utmost importance, it is necessary to use a model that begins with the smallest window and works outwards, testing increasingly larger windows, until it detects a slope change associated with a boundary between two land cover regions. A sharp increase in the slope of the SDTP curve generally marks the beginning of the boundary between two land cover regions, and thus, is the largest window that does not overlap the boundary. This is illustrated in Figure 2. 


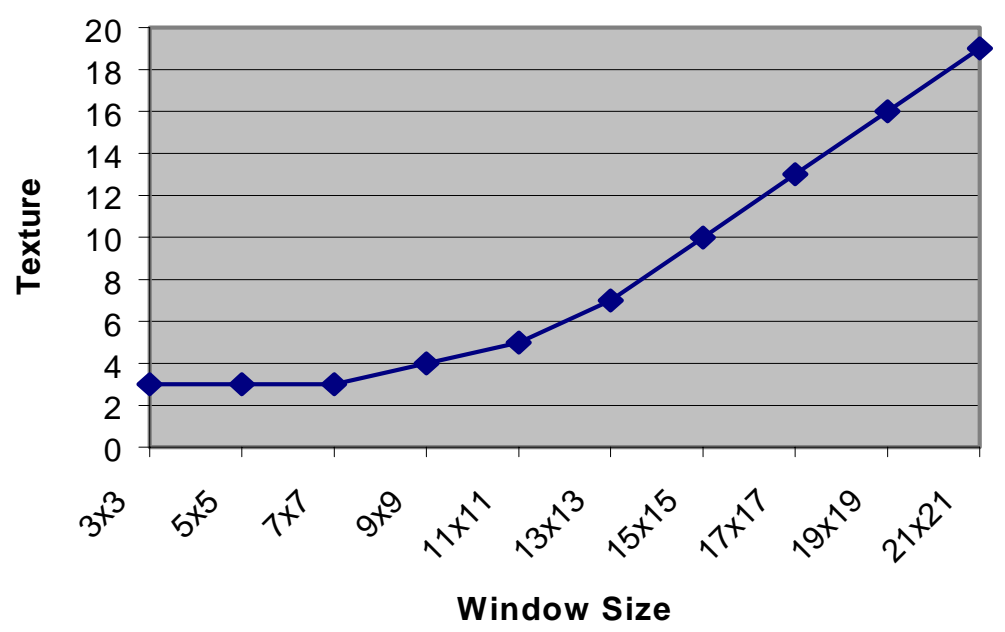

Figure 2: Land cover boundary on SDTP curve.

Figure 2 is a chart of an SDTP curve. The slope remains constant out to the $7 \times 7$ window, where it begins to rise. This is marked increase in slope represents the boundary between two land cover regions.

A slope that is neither increasing nor decreasing, or is changing very little, indicates that neighboring pixels are nearly the same texture as the pixel of interest. These neighboring pixels most likely belong to the same land cover region as the pixel of interest. Since it is desirable to use as large a window as possible, the model will continue to test increasingly larger windows until it reaches an inflection point.

A slope that is decreasing indicates that the center pixel is located on a relatively small object that has contrasting DN values compared to the area surrounding it. An example of this might be if the pixel of interest lies on a house or small field that is surrounded by forest. As the window sizes get larger, the relatively homogenous forest pixels begin to dominate the standard deviation measurement. Since the object is so 
small, it is not large enough to be considered a land cover region by itself, and would therefore be considered as within-class variability. It would therefore be preferable to use larger windows until the slope of the SDTP curve begins to increase. This would indicate that a new land cover boundary has been reached.
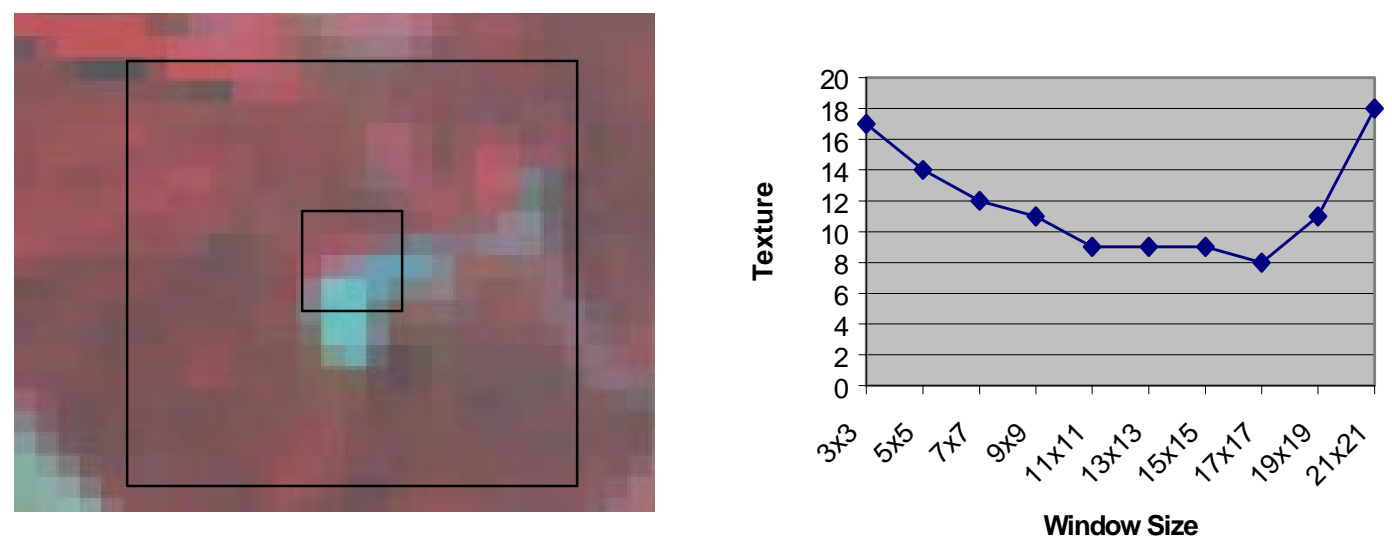

Figure 3: Left: SPOT False Color Composite Image of a building with rectangles representing $5 \times 5$ and $21 \times 21$ windows. Right: SDTP curve for the pixel in the center of the rectangles in left image. Note the decreasing local texture in smaller windows.

Figure 3 illustrates how an object in H-Resolution such as this small, built area can have a high texture value. The small rectangle represents the $5 \times 5$ window. This window contains a relatively even amount of built pixels and forest pixels, yielding a texture value of 17 . This built object is too small to be considered as a separate land cover region. As window size is increased, the SDTP curve continues to drop due to the increasing proportion of forest pixels in the larger windows. Finally, a minimum point is reached at the $17 \times 17$ window, and the SDTP slope increases for larger windows. The largest rectangle represents the $21 \times 21$ window. This window has a wide range of cover types, including a grassy area, thus causing a drastic rise in the texture value. The 
minimum value, in this case the $17 \times 17$ window, is the optimal size window to use in this scenario.

An increasing slope indicates that a large amount of variability exists around the pixel of interest. If the slope rises immediately from the smallest window, it is best to search for larger texture windows along the SDTP curve until a slope segment is reached where there is little change. This would indicate that the largest windows have finally reached a point where the texture is not changing. The point where the slope begins to rise again from this plateau in the SDTP curve generally marks the beginning of a new land cover boundary. The window at that point would be the optimal size window to use in that case.
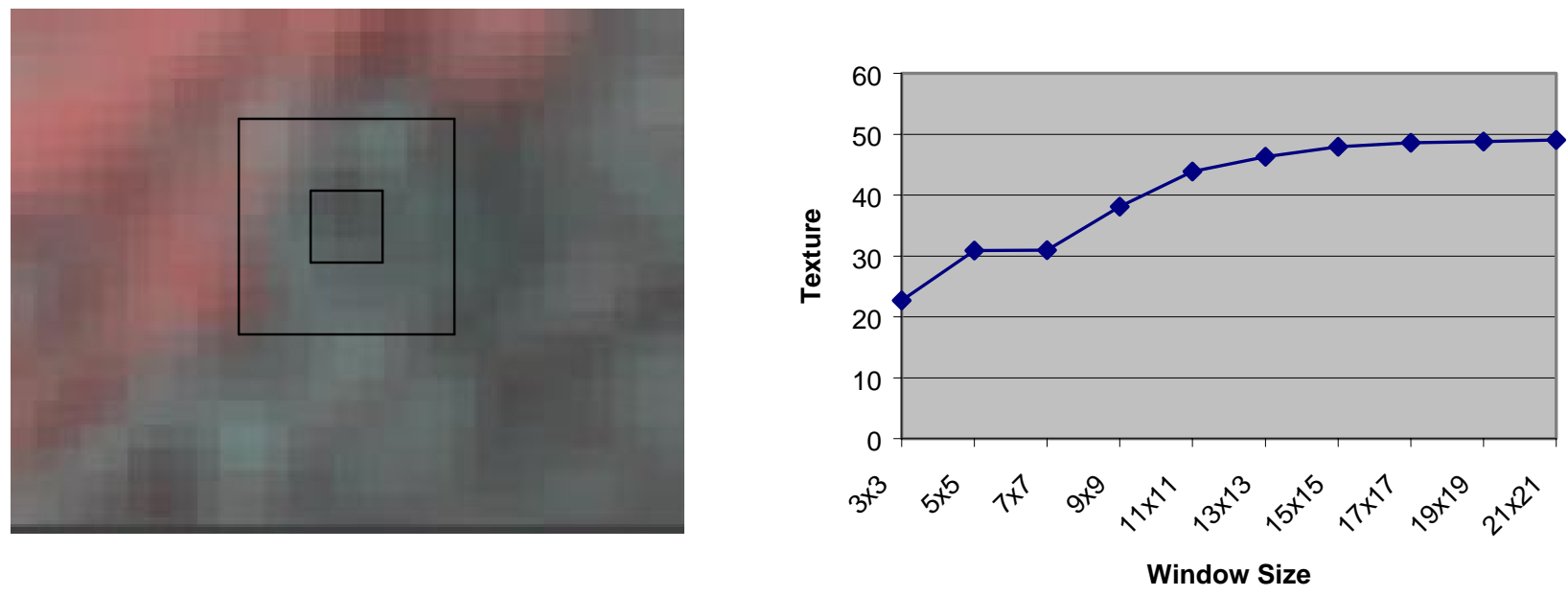

Figure 4: Left: ADAR False Color Composite Image of Shaded Forest class. Right: SDTP curve for the pixel located in the center of the rectangles in the image on the left.

Figure 4 is an example of a pixel that lies in an area of increasing texture associated with larger windows. The $3 \times 3$ window (represented by the smaller rectangle) contains mostly shadow pixels from nearby trees. The outer rows of the $5 \times 5$ window 
contain some tree pixels, represented by the lighter shades. The combination of the trees and their shadows lead to higher texture values for the $5 \times 5$ and $7 \times 7$ windows, and are a form of within-class texture. The 9x9 window detects pixels from the land cover class Grass (represented by the red shades), causing a further increase in the texture. In this case, the optimal size window model would use the $7 \times 7$ window since it represents the largest window that measures within-class texture without measuring between-class texture.

If the slope between the first and second windows is constant, then the pixel lies on an object that is large enough to be considered as a distinct land cover region. The model continues to test larger windows until a slope segment increases significantly. The window size at that point of increase would be the optimal size window to use in that scenario. This is illustrated in Figure 5.
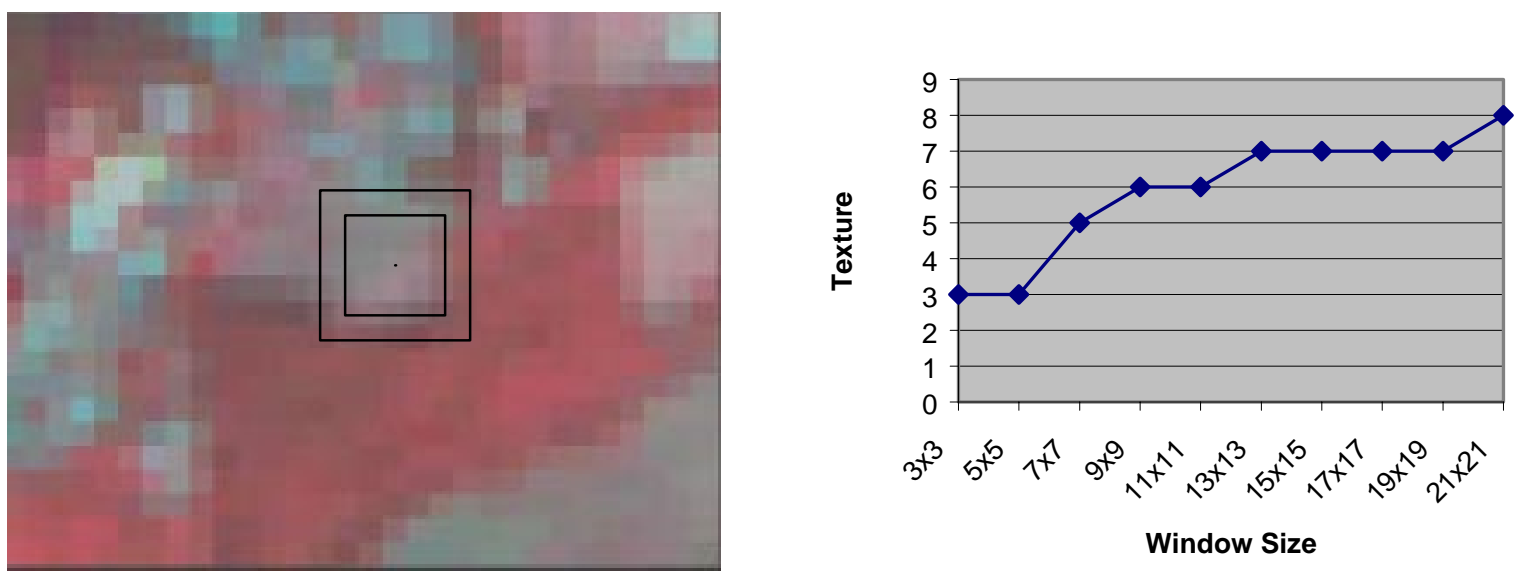

Figure 5: Left: SPOT image of Residential area along the boundary of a forested area. Smaller rectangle represents a 5x5 window and larger rectangle represents a 7x7 window. Right: SDTP curve of pixel at center of rectangle in left image, showing sharp increase in the slope of SDTP curve between two windows in right image. 
In Figure 5, the pixel of interest lies in a Residential area composed of mixed pixels with houses, trees, and yards. The smaller rectangle indicates the dimensions of the $5 \times 5$ window, which is entirely made up of mixed pixels. The $3 \times 3$ window, which is centered within the $5 \times 5$ window has a similar composition of mixed pixels. Thus, the slope remains flat on the SDTP curve between the $3 \times 3$ and $5 \times 5$ windows. However, the slope begins to rise between the $5 \times 5$ window and the $7 \times 7$ window, which is represented by the larger rectangle in the image. The outer rows of the $7 \mathrm{x} 7$ window include pure forest pixels. These forest pixels have much higher DN values, causing the texture value to rise from 3 to 5 . Therefore, the $5 \times 5$ window would represent the largest window before a sharp increase, and would be the optimal size window in this case.

Detecting these changes in the texture between two land cover regions forms the basis of the Optimal Size Window Model. Rules were coded in the Imagine Spatial Modeler, hereafter referred to as qualifying statements, to determine optimal size window selection in the model.

Table 2: Rules for the Optimal Size Window Model

1. IF MAXIMUM VALUE WINDOW - 3 X 3 WINDOW > 3 * (3 X 3 WINDOW), THEN USE $3 X 3$ WINDOW, OTHERWISE GO TO NEXT RULE.

2. IF 5 X 5 WINDOW - 3 X 3 WINDOW $<0.25 *$ ( 7 X 7 WINDOW - 3 X 3 WINDOW), THEN USE 5 X 5 WINDOW, OTHERWISE GO TO NEXT RULE.

3. IF 7 X 7 WINDOW - 5 X 5 WINDOW $<0.25 *$ ( 9 X 9 WINDOW - 5 X 5 WINDOW), THEN USE 7 X 7 WINDOW, OTHERWISE GO TO NEXT RULE.

4. IF 9 X 9 WINDOW -7 X 7 WINDOW $<0.25 *(11$ X 11 WINDOW - 7 X 7 WINDOW), THEN USE 9 X 9 WINDOW, OTHERWISE GO TO NEXT RULE.

5. IF 11 X 11 WINDOW - 9 X 9 WINDOW < $0.25 *(13$ X 13 WINDOW - 9 X 9 WINDOW), THEN USE 11 X 11 WINDOW, OTHERWISE GO TO NEXT RULE. 
6. IF $13 \mathrm{X} 13$ WINDOW - $11 \mathrm{X} 11$ WINDOW < $0.25 *(15 \mathrm{X} 15$ WINDOW - $11 \mathrm{X} 11$ WINDOW), THEN USE 13 X 13 WINDOW, OTHERWISE GO TO NEXT RULE.

7. IF 15 X 15 WINDOW - 13 X 13 WINDOW $<0.25 *(17$ X 17 WINDOW - 13 X 13 WINDOW), THEN USE 15 X 15 WINDOW, OTHERWISE GO TO NEXT RULE.

8. IF 17 X 17 WINDOW - 15 X 15 WINDOW < $0.25 *(19$ X 19 WINDOW - 15 X 15 WINDOW), THEN USE 17 X 17 WINDOW, OTHERWISE GO TO NEXT RULE.

9. IF 19 X 19 WINDOW - 17 X 17 WINDOW < $0.25 *$ ( 21 X 21 WINDOW - 17 X 17 WINDOW), THEN USE 19 X 19 WINDOW, OTHERWISE USE 21 X21 WINDOW.

\section{Dynamic Range Test}

Rule Number 1 is termed the Dynamic Range Test. It is a special case rule that applies only to cases where there was an extreme range between the smallest and largest windows. Land cover regions with very low DN values such as water can have boundary gradients with other land cover regions that are very steep. These result in very abrupt changes in the slope of the SDTP curve where the larger windows overlap onto neighboring land cover regions with higher DN values. These abrupt rises in the slope are easily detectable through a Dynamic Range Test, in which the smallest window (3x3) is subtracted from the highest value of any window in the layerstack, represented by the Maximum Value Window. It was found empirically that a difference between the Maximum Value Window and the $3 \times 3$ window that was three times greater than the value for the $3 \times 3$ window was a good discriminant of these boundaries.

If the pixel passed this dynamic range test, then it must have an extremely low value for its $3 \times 3$ window and extremely high values in the larger windows, thus signifying that it must be a member of a region characterized by low $\mathrm{DN}$ values. In all other cases, the goal of the model would have been to use the largest window that was 
possible before the boundary for a new land cover region was reached. However, in this case, since the region had such low texture, any window, including the smallest ( $3 \times 3)$, that lies entirely within its boundaries would produce a texture value that represents the entire region. Using the $3 \times 3$ window maintained accuracy while it reduced the computational task of the model.
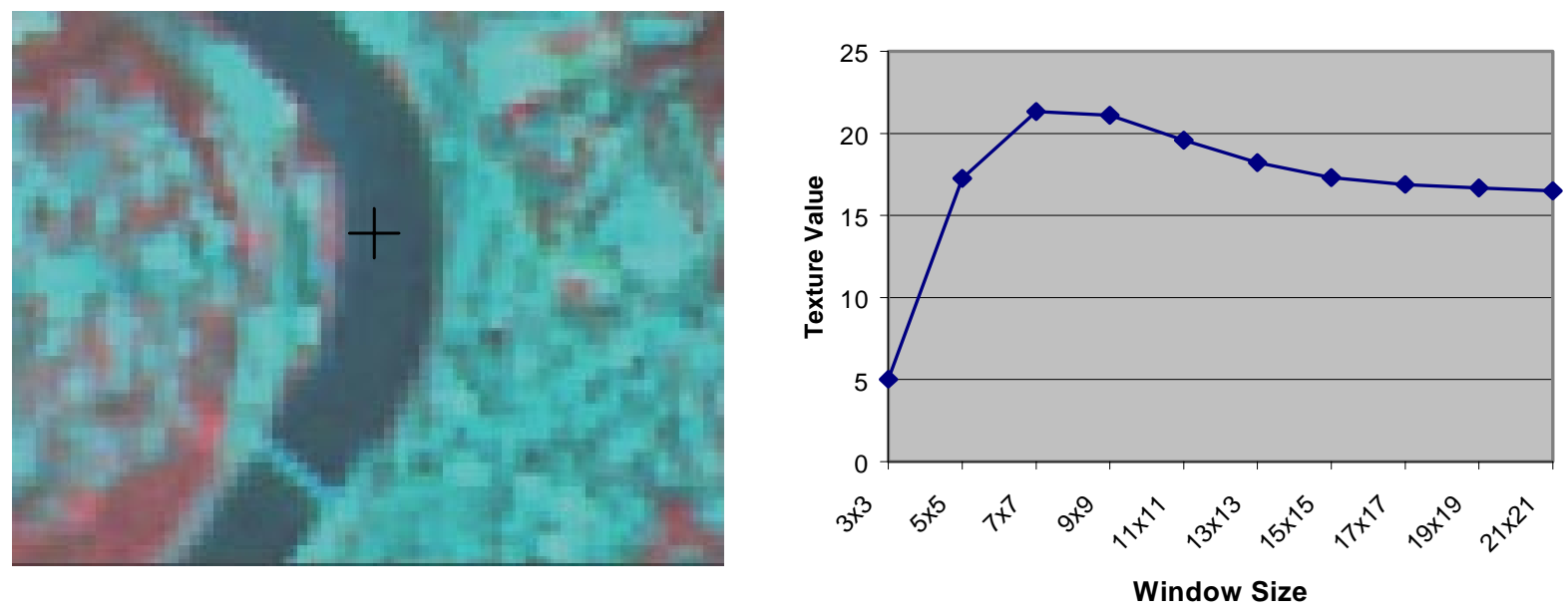

Figure 6: Left: SPOT image of the Monongahela River, Morgantown, WV. Crosshairs represent pixel located two pixel lengths from left shoreline. Right: SDTP curve for the pixel marked in the image on the right. This is an example of the large dynamic range scenario.

The SDTP curve illustrated in Figure 6 is an example of the large dynamic range between the highest texture value and the value for the $3 \times 3$ window. The outer left column of the $3 \times 3$ window contains some mixed pixels that are associated with the shoreline, giving it a texture value of 5 . The $7 \times 7$ window contains a large number of built and forested pixels in addition to the water pixels. This highly varied combination of pixels results in a very high texture value of 22 . Since 22 is over 3 times greater than 5, the Optimal Size Window Program would use the $3 \times 3$ window in this case. 


\section{TESTS OF LOCAL SDTP SLOPE CHANGE}

A central issue is to determine the size of the window that best captures the texture of a region without overlapping a neighboring land cover region. As mentioned previously, a major increase in the slope of the SDTP curve tends to indicate the presence of a boundary between two land cover regions. A rapidly increasing or decreasing slope in the direction of increasing window size may indicate that the local texture at this location is highly varied and that a larger window is needed to capture the "true" texture of the region. Once a major change in slope is detected, the model would use the window that is the largest size before the change occurs. To determine these points, each slope segment between two window sizes must be measured to determine the slope. This can be accomplished by subtracting the texture value of the smaller window from the next largest one.

Next, a method for comparing the slope value for this segment of interest to other segments must be established. Since one of the goals of the Optimal Size Window Program is to develop a model that is robust enough to use across different types and scales of images, a predetermined, generic slope value can not be specified. Slope values for segments from images of different areas and scales are likely to differ substantially.

To overcome this problem, the slope of segments of the SDTP curve can be compared to the slope of neighboring segments within the same SDTP curve. However, comparing the slope of one segment with the next segment of the next larger pair of windows on the curve would not necessarily always detect a change. As mentioned previously, the SDTP curve tends to be asymptotic at the larger window sizes. Thus, small changes in slope are less significant with the smaller windows. Not accounting for this issue would pose a significant threat to the internal validity of the model. 
It is therefore preferable to identify slope changes based on a comparison of the slope of each segment with the average slope of that segment and the next larger segment immediately adjacent to the segment of the SDTP curve. By comparing a particular segment with its local average, the effect of the asymptotic relationships at larger window sizes can be minimized. For example, if an abrupt change does occur, with the slope of one segment being positive and the next being negative, it will be reflected in a low slope value for the local average. Thus, a difference in the slope value for a particular segment and the slope value for the local average would tend to indicate the presence of a boundary between two land cover regions of differing texture.

Two nominal categories were defined, based on an arbitrary threshold: one indicating that a change in direction of the slope had taken place, and the other indicating that it had not. The threshold was calculated as a percentage of the local average. A threshold value that is too low may allow the model to select large windows that overlap boundaries between two or more desired land cover regions. A value that is too high risks being overly sensitive to slight changes in texture, and would confine the model to using windows that may be too small to accurately represent a particular land cover region's texture. A qualitative assessment using samples from throughout each of the three images found that a threshold value of 25 percent of local texture proved sufficient for selecting optimal window size based on land cover region boundaries. 

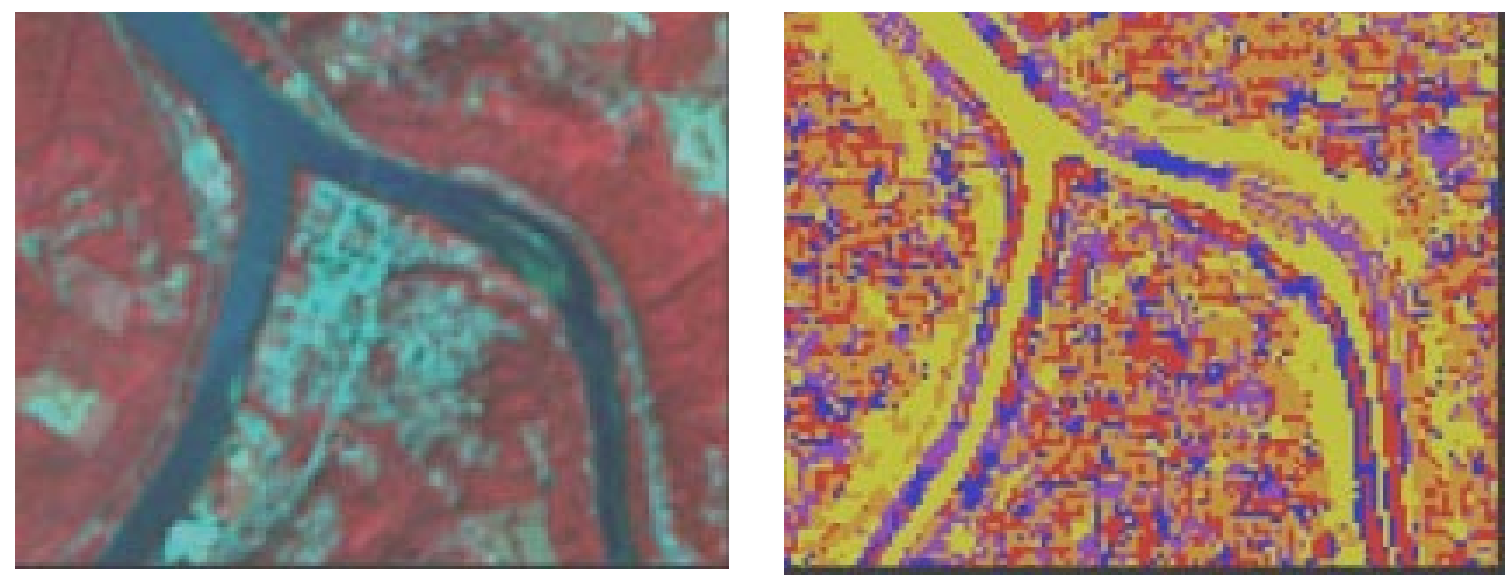

Figure 7: Left: SPOT image of the confluences of the Monongahela and Cheat Rivers. Right: Optimal Size Windows selected by the Optimal Size Window Program for the same region as the image on the left. Key: Yellow $=3 \times 3,5 \times 5$ windows, Orange $=7 \times 7$, $9 \times 9$ windows, $\operatorname{Red}=11 \times 11,13 \times 13$ windows, Purple $=15 \times 15,17 \times 17$ windows, Blue $=19 \times 19,21 \times 21$ windows.

Figure 7 illustrates how the application of the rules to determine the optimum window size. A SPOT 20m pixel image is on the left and an image of the optimal sized window calculated by the model with a threshold value of a 25 percent change in slope, on the right. One notable relationship between the two images is the use of the smallest $3 \times 3$ (yellow) windows in the rivers. This is an excellent example of the success of the Dynamic Range Test. The smallest windows are contained within the boundaries of the river while the larger windows overlap onto neighboring land cover regions producing a very large dynamic range in the texture values of the SDTP curve.

If the segment of interest was less than 25 percent of the local neighborhood segment, then this indicated the larger window of the segment of interest probably incorporates two land cover regions. Thus, the window size in the middle of the local neighborhood of window sizes would be the "optimal" sized window. It is inferred that this window would contain the maximum amount of sample pixels without overlapping 
two different land cover regions, and most likely lies on an H-Resolution object that is large enough to be classified as a land cover region.

If the slope of the segment of interest was greater than $25 \%$ of the local neighborhood segment, then this indicated that a great deal of difference in the local texture exists between these two windows. Therefore, it was deduced that the pixel did not lie on an H-Resolution object that was at least 25 pixels in area. For this research, any region that was not at least 25 pixels in area was deemed too small to consider as a separate land cover region. In other words, these regions that are less than 25 pixels in area were considered as a constituent of the local texture of the image. Therefore, in these cases, a larger window was needed to smooth out this local, within-class variation in order to provide an accurate measure of texture. While the inclination might be to use the larger of the two windows ( $5 \times 5$ window) in this segment, a great deal of variation may exist between this window and an even larger window $(7 \times 7)$ due to the low value of the threshold percentage. Therefore, the model moves on to a new test that compares the slope of that segment ( $7 \times 7$ window $-5 \times 5$ window) with its corresponding local neighborhood slope (9x9 window $-5 \times 5$ window). Thus, the model moves from left to right (i.e. smaller to larger windows) until a segment that is less than the threshold value is detected. If all of the values, including the 19x19 window - 17x17 window segment, are greater than the threshold, then the $21 \times 21$ window is used because there are no larger windows in the model.

\section{Adaptive Minimum Window Program}

If a large dynamic range of $\mathrm{DN}$ values exists in the average brightness between two land cover regions, a very steep texture gradient can occur. In cases where the pixel of interest lies directly adjacent to this boundary, even the smallest window (3x3) will 
contain pixels from both land cover regions. This results in an SDTP curve that has unusually high values for all windows. The Dynamic Range Test of the Optimal Size Window Program can fail to detect this situation because the slope analysis is based on relative values. Under this circumstance, even the $3 \times 3$ window is too large.

An adaptive window, which uses the lowest value in a local window of texture pixels, is an effective method of minimizing problems of this nature. Creation of this adaptive window is a two step process, involving a separate $3 \times 3$ texture channel followed by a $3 \times 3$ minimum value window of the $3 \times 3$ texture in the same model. This new adaptive window channel can then be added to the other texture images using the Spatial Modeler command "STACKLAYERS". A new Dynamic Range Test, similar to the one used in the Optimal Size Window Program, can be used to detect circumstances when a pixel lies on an extremely steep boundary gradient. Due to the fact that in many cases, a very large dynamic range between the highest value in the SDTP curve and the $3 \times 3$ Adaptive Minimum Window will exist in these extreme boundary gradients, some method must be used to delineate circumstances when the adaptive window will be used, and when the conventional $3 \times 3$ window will be used. In most cases when the $3 \times 3$ Adaptive Minimum Window would best be used, the dynamic range between the highest value on the SDTP curve and the $3 \times 3$ window will be less than 3 times the value for the $3 \times 3$ window. Yet, the dynamic range between the highest value on the SDTP curve and the $3 \times 3$ Adaptive Minimum Window would still be very high. This test was then added as a separate line to the Optimal Size Window Model to create the Adaptive Minimum Window Model: 
IF Maximum Value Window - 3x3 Window $<3$ (3x3 Window) and $3 \times 3$ Window - 3x3 Adaptive Window > 0.67 ( $3 \times 3$ Window)

THEN Optimal Texture $=3 \times 3$ Adaptive Window 


\section{CHAPTER SIX $\underline{\text { RESULTS }}$}

The results of this thesis have been presented sequentially by data type. For each data type, a visual comparison between each classified image and an aerial photograph was used to assess qualitatively the general accuracy of the classification. This section is then followed by a discussion of classification error matrices, with results for edge, interior, and edge plus interior, presented in a particular order. First, an assessment of the

overall accuracy, including the highest and lowest Kappa Indices of Agreement (KIA) for all of the images for a particular type of test data are presented. Next, any land cover classes that are similar in accuracy throughout all of the images are listed. This is followed by any observed differences for a particular land cover type throughout the images. For example, if a land cover has high KIAs in one image and low KIAs in another, it will be discussed. Finally, an overall assessment is presented to determine if any trends exist in the error matrices. Once both the qualitative and quantitative assessments have been presented, they are tied together in an overall assessment.

After the accuracy of all three data types have been assessed, the results will be analyzed to determine if the use of optimal size and adaptive minimum windows improved the accuracy of the classifications.

\section{$\underline{\text { SPOT Accuracy Assessment }}$}

The accuracy of the SPOT image classified using only the spectral data was very

high. However, some errors did exist, especially along edges where Forest regions were bordered by Water and Coal/Mining. In some cases scattered throughout the image, 
these areas were misclassified as Residential. This may have been due to the large number of mixed pixels that were selected in the training data for the Residential class. Many of the border areas contained mixed pixels that were spectrally similar to the mixed pixels in the Residential class. Another minor source of error was in the misclassification of some Residential areas such as streets, as Sparse Grass. Finally, some Water was classified as Coal/Mining or Urban/Barren, and some Urban/Barren was classified as Coal/Mining.

The classified image incorporating $3 \times 3$ texture in addition to the spectral bands was nearly indistinguishable from the image using only spectral data. However, there was a slight decrease in accuracy in some of the edge areas due to increased misclassification of Residential.

The image incorporating the $11 \times 11$ texture was slightly more accurate than the spectral image and the $3 \times 3$ image. The error due to edge and Grass areas being classified as Residential was significantly reduced. However, a five pixel wide area of error existed along the outer edge of the entire image. This was an artifact of the Imagine texture calculation program, which does not compensate for windows that extend beyond the scene edge.

The overall accuracy of the image incorporating the $21 \times 21$ texture was nearly the same as the 11x11 image. However, in some cases, Sparse Grass was classified as Urban/Barren, and Residential was misclassified as Grass/Crops. This was especially true along the edges of the Forest/Residential boundaries. Also, there was a greater amount of error due to Water being classified as Coal/Mining. There was a 10 pixel zone of error around the outer boundary of the image. 
The classification results of the image incorporating the optimal size model were an improvement over all of the other images. The most notable improvement in accuracy came from the elimination of errors due to pixels along the Forest/Residential boundary that had been classified as Grass/Crops. This had been a major problem in the classification of fixed size texture images. Also, there was a great reduction in the amount of error caused by Sparse Grass being misclassified as Urban/Barren. This was especially true near the airport. Finally, there was a noticeable reduction in the amount of error near the outer boundaries of the image.

Surprisingly, the classified image incorporating the optimal size model with the adaptive minimum window was not as accurate as the image incorporating only the optimal size model. A type of error that existed in this image, but was not present in the optimal size model image, was where the edges of Water and Coal/Mining were misclassified as Urban/Barren. This may have been due to the high texture values along these edges being replaced with low texture values by the adaptive minimum window. Although this image was not as accurate as the optimal size window image, it was still an improvement in accuracy over all of the others.

Table 3: SPOT Error Matrices (Overall Kappa Indices of Agreement)

\begin{tabular}{|l|c|c|c|}
\hline & $\begin{array}{c}\text { INTERIOR + } \\
\text { EDGES }\end{array}$ & EDGES & INTERIOR \\
\hline SPECTRAL & 0.88 & 0.87 & 0.92 \\
SPECTRAL + 3 X 3 & 0.89 & 0.86 & 0.93 \\
SPECTRAL + 21 X 21 & 0.87 & 0.84 & 0.87 \\
SPECTRAL + OPTIMAL SIZE & 0.82 & 0.87 & 0.70 \\
SPECTRAL + OPTIMAL & 0.89 & 0.87 & 0.92 \\
SIZE/ADAPTIVE MINIMUM & 0.89 & 0.85 & 0.93 \\
VALUE & & & \\
\hline
\end{tabular}


Results from the error matrices were statistically significant, and indicate that the overall accuracy for the SPOT data was high. The overall KIA for the test data that was selected from throughout each land cover region was high, ranging from 0.82 for the $21 \times 21$ image to 0.89 for the image incorporating the adaptive minimum window. The Water and Forest classes had high KIAs in all of the images. Urban/Barren had the lowest KIAs throughout all of the images.

The classification of pixels near edges of classes produced KIAs in the 0.80 range, a result slightly lower than for the test pixels incorporating both edges and interior pixels. The Water, Forest, and Grass/Crops classes all had high KIAs. Sparse Grass had high KIAs in all of the images except the image incorporating the $21 \mathrm{x} 21$ texture. This may have been due to the small size of the Sparse Grass regions. The 21x21 window overlapped two or more land cover regions, creating an unrepresentatively high texture value. The Urban/Barren class had the lowest KIAs throughout all of the images. However, there was some improvement when comparing the spectral image with the images that incorporated texture for this class. As the window size gets larger, the KIA for the Urban/Barren tends to increase. Yet, overall KIA for the 21x21 window was low at 0.70 . This seems to indicate that the larger windows were better at classifying the within-class variability than the smaller windows or the spectral information alone. The low KIA in the $21 \times 21$ seems to indicate that these windows overlapped onto other land cover regions, creating greater between-class variability. The image incorporating the 
optimal size texture had the highest KIA for the Urban/Barren class for both producer's 0.76 and user's 0.60 accuracy.

The KIAs for the test data selected from the interiors of classes were very high, with the exception of the $21 \times 21$ image. A significant portion of this error was due to Water being misclassified as Urban/Barren. Because both of these classes were very low-textured and the test data was selected from the interiors of classes (eliminating the between-class variability), these two classes were not very separable. This was actually a rare instance where the between-class variability may have actually increased the separability of the two classes.

Judging from both the qualitative assessment, and the error matrices, it appeared that the addition of texture did improve classification accuracy classes that contained high within-class variability and large numbers of mixed pixels. However, while the images that incorporated the largest windows improved accuracy due to the better characterization of within-class texture, errors due to between-class texture became a problem. This apparent contradiction appeared to be at least partially resolved by the images incorporating the optimal size windows, and combination optimal size/adaptive minimum texture windows. The classified images that incorporated the optimal size texture, and the combination optimal size and adaptive texture appeared to be the most accurate. However, in a few cases, the image incorporating the combination optimal size/adaptive minimum texture may have actually reduced accuracy by replacing unusually high texture values in edge pixels with artificially low values.

\section{SIR-C Accuracy Assessment}

The qualitative evaluation of the SIR-C image using only the two spectral bands was moderate to poor. Many areas of Forest were misclassified as Residential throughout 
the image. Also, some Residential areas were misclassified as Forest. Areas of Forest that were in terrain shadows were misclassified as Grass. Some Urban areas were misclassified as Residential and Forest. The edges of Flat Surfaces (roads and water bodies) facing the radar antenna acted as corner reflectors, thereby producing an unusually strong radar return that was misclassified as Urban.

The accuracy of the images incorporating texture from fixed size windows was not substantially different from the image using only the spectral data. However, there was a very slight reduction in the error of Forest being misclassified as Residential, especially in the data set incorporating the $21 \times 21$ window.

The image incorporating the optimal size texture offered a major improvement in accuracy. However, the edges of the Water class misclassified as Urban was much more pronounced in this image. Also, more Forest was misclassified as Residential. However, there was less error due to Residential being misclassified as Forest as in the other images. One of the major improvements in this image compared with the other images was a decrease in the amount of Forest in terrain shadows being misclassified as Grass.

The image created by adding the adaptive minimum window to the optimal size texture was even more accurate. There was less error due to Forest being misclassified as Residential, less Water misclassified as Urban, and less Urban was misclassified as Residential. It appears from these improvements in accuracy that the use of the adaptive minimum window to replace the unusually high values along the edges with the minimum value was successful. 
Table 4: SIR-C Overall Kappa Indices of Agreement

\begin{tabular}{|l|c|c|c|}
\hline & $\begin{array}{c}\text { INTERIOR + } \\
\text { EDGE }\end{array}$ & EDGE & INTERIOR \\
\hline SPECTRAL & 0.51 & 0.41 & 0.48 \\
SPECTRAL + 3 X 3 & 0.51 & 0.41 & 0.46 \\
SPECTRAL + 11 X 11 & 0.51 & 0.46 & 0.45 \\
SPECTRAL + OPTIMAL SIZE & 0.48 & 0.41 & 0.46 \\
$\begin{array}{l}\text { SPECTRAL + OPTIMAL } \\
\text { SIZE/ADAPTIVE MINIMUM } \\
\text { VALUE }\end{array}$ & 0.71 & 0.50 & 0.60 \\
\hline
\end{tabular}

The KIAs for the error matrices were statistically significant with low to moderate accuracy when comparing the classified images with the test data taken from both edges and interiors. These KIAs ranged from 0.48 for the $21 \times 21$ texture image to 0.71 for the optimal size texture image. The KIA for the image only incorporating spectral data was 0.50. The Shadow/Flat Surfaces class had the highest KIAs throughout all of the data sets, while the Urban and Residential classes had the lowest KIAs. However, there were significant improvements in the KIAs for Forest, Urban, and Residential when comparing the images incorporating the optimal size and combination optimal size/adaptive minimum texture with the other images.

The error matrices for the edge test data had low KIAs, ranging from 0.41 for the image incorporating only the spectral information to 0.50 for the image incorporating the optimal size texture. Again, the Shadow/Flat Surfaces class had the highest KIA throughout all of the images. The Residential class had very low KIAs throughout all of the images. The Grass class had low KIAs for the spectral image, but its KIAs increased when using the fixed size texture bands. The optimal size texture image had the highest KIA for Grass. The Users accuracy for the Forest class was much higher for the optimal 
size texture image than for the spectral and fixed size texture images. The image incorporating the combination of optimal size/adaptive texture had a very low user's KIA for Grass, and low producer's and user's KIA for Residential.

The overall KIA for the error matrix for the test data taken from the interiors of land cover regions was low to moderate, ranging from 0.45 for the $11 \times 11$ to 0.72 for the image incorporating the combination of optimal size/adaptive minimum texture. The overall KIA for the spectral image was 0.48 , and 0.71 for the optimal size texture image. Once more, the Shadow/Flat Surfaces class had the highest KIA throughout all of the images. The Forest class was low throughout, with low producer's and high user's KIAs. The optimal size texture image and the combination optimal size/adaptive minimum texture image had much higher KIAs for the Urban, Residential, and Grass classes.

The results of the qualitative assessment and the error matrices seem to suggest that the incorporation of texture with spectral data can improve classification accuracy for the radar data. This may have been due to the high variability of the SIR-C data, resulting from the radar speckle. This high frequency noise created multi-modal distributions for spectral classes, thus decreasing the separability between these classes. However, the incorporation of texture appears to have created grey-level values that were spectrally more separable than the spectral data alone. The largest windows best characterized the within-class texture, but exacerbated the error due to between-class variability. This is illustrated in the error matrices for the test data selected from the class edges. The KIA for the $3 \times 3$ was 0.41 , which was similar to the KIA for the spectral data alone. This window size was too small to characterize the within-class variability and increase the separability between classes. The KIA for the $11 \mathrm{x} 11$ was an improvement at 
0.46. Finally, the KIA for the $21 \times 21$ dropped to 0.43 due to the increase in between-class variability. The $11 \times 11$ window was a compromise between characterizing the withinclass variability and creating error due to between-class variability. The optimal size window was an even better compromise. By using the $3 \times 3$ window near the extreme edges, it was able to reduce the between-class error even further, while maximizing the characterization of the within-class variability to achieve a KIA of 0.50 . The image incorporating the combination of the optimal size/adaptive minimum texture was less successful on the edges. As with the SPOT data, it may have overcompensated by replacing unusually high values created by between-class variability with artificially low values.

\section{$\underline{\text { ADAR Accuracy Assessment }}$}

Overall, the accuracy of the classified ADAR images was very high. Using the qualitative assessment, very few errors were noticeable, especially in the image classified using only the spectral data. Several very minor errors in the spectral image included: Water misclassified as Rooftop, Rooftop misclassified as Concrete, Grass and Barren misclassified as Sunlit Forest, and Astroturf misclassified as Concrete. Most of these errors were due to the classes being spectrally similar, and therefore very difficult to separate. However, one example of error due to within-class variability was in the Shaded Forest being misclassified as Rooftop.

The fixed size texture images were less accurate then the spectral image. As the windows became larger, the errors due to between-class edge effect increased. This was especially true where Grass was misclassified as Rooftop. One notable exception to this 
trend was in the $21 \times 21$ image, where much less Shaded Forest was misclassified as Rooftop, as in the other images. In this case, the large sample size of the $21 \times 21$ window was sufficient to generate a relatively uniform texture for the trees and shadows.

However, the large size of the $21 \times 21$ window also caused edge effect errors where Water was misclassified as Concrete. The optimal size image was similar, though not quite as accurate as the spectral image. It appears that, while the optimal size program overcame most of the edge effect errors of the fixed windows, a few still existed. The combination optimal size/adaptive minimum window was slightly more accurate than the optimal size alone. However, it was still not as accurate as the spectral image.

Table 5: ADAR Overall Kappa Indices of Agreement

\begin{tabular}{|l|c|c|c|}
\hline & $\begin{array}{c}\text { INTERIOR + } \\
\text { EDGE }\end{array}$ & EDGE & INTERIOR \\
\hline SPECTRAL & 0.93 & 0.87 & 0.90 \\
SPECTRAL + 3 X 3 & 0.93 & 0.86 & 0.90 \\
SPECTRAL + 11 X 11 & 0.92 & 0.84 & 0.90 \\
SPECTRAL + 21 X 21 & 0.91 & 0.78 & 0.92 \\
SPECTRAL + OPTIMAL SIZE & 0.93 & 0.86 & 0.90 \\
SPECTRAL + OPTIMAL & 0.93 & 0.87 & 0.90 \\
SIZE/ADAPTIVE MINIMUM & & & \\
VALUE & & & \\
\hline
\end{tabular}

The error matrices of the test data taken from combined edges and interiors test data set had very high KIAs in the lower 0.90 range. All of the KIAs for the ADAR data were statistically significant. The lowest KIA was the $21 \times 21$ image at 0.91 . The users' accuracy of the Rooftop class was highest in the spectral image, but dropped in the 
texture images as window size increased. This may have been due to the small relative size of the individual rooftops. The $21 \times 21$ windows overlapped onto other land cover regions. The KIAs of Grass and Shaded Forest was also highest in the spectral image and lowest in the $21 \times 21$ image. Conversely, the $21 \times 21$ image had the highest KIA for Sunlit Forest while the spectral image had the lowest.

The overall KIAs for the error matrices created from test data taken from edges was lower than for the data taken from the edges and interiors. The spectral image had the highest KIA at 0.87 while the $21 \times 21$ had the lowest at 0.78 . In nearly all classes, the KIAs were highest in the spectral image and lowest in the $21 \times 21$ image. Again, the exception was in the Sunlit Forest class.

The 21x21 texture image had the highest overall KIA for the test data taken from the interiors of land cover regions. This may have been due to the ability of the large windows to obtain large samples in the Sunlit and Shaded Forest classes. The substantial between-class errors in the $21 \times 21$ image were not as dominant as in the test data taken from edges, and combination edges and interiors.

The results of the qualitative and quantitative assessments for the ADAR data indicate that the addition of texture to the spectral data rarely improved accuracy, and in some cases, actually decreased it. This was due to the relatively small size of the pixels when compared to objects on the ground. Most objects were in H-Resolution, and were dominated by low tonal contrasts. The only texture in the image was in the Sunlit and Shaded Forest classes. In the Sunlit Forest class, the individual sunlit trees contrasted sharply with their shadows, creating a low frequency, high contrast texture. In this particular case, the $21 \times 21$ texture image was superior in accuracy. However in most 
other cases, the addition of texture created large edge effect errors along the land cover region boundaries. The optimal size and optimal size/minimum value windows were partially effective at eliminating this error. 


\section{CHAPTER SEVEN CONCLUSIONS}

The results of this research can be summarized as follows:

1. The incorporation of texture with spectral data can improve classification accuracy in land cover regions with high, within-class variability.

2. Texture measured from large, fixed size geometric windows in many cases reduced accuracy by introducing substantial, between-class, edge effect errors.

3. The optimal size and the optimal size/minimum-value windows improved accuracy by characterizing within-class variability while reducing between-class, edge effect errors in the radar data, but had mixed success in the ADAR and SPOT data.

Classifiers using only spectral data can produce substantial errors in land cover regions that contain high within-class variability. The incorporation of texture can improve classification accuracy in these regions by upscaling the regularization process, thus producing a region with low tonal contrasts. In the SPOT images, the image classified with only spectral data misclassified Grass/Crops and the edges of Forest as Residential. This error was reduced in the images incorporating texture as window size increased. In the ADAR images, the $21 \times 21$ texture image reduced the within-class error in the Sunlit Forest class. Finally, the most observable improvement in within-class accuracy due to texture was in the SIR-C data. In the spectral image, Forest was misclassified as Residential. This was reduced with the incorporation of texture. 
While texture from large, fixed size windows reduced within-class accuracy, in many cases it created substantial errors due to between-class edge effects. An example of this was in the SPOT images, where Sparse Grass was misclassified as Urban/Barren and the edges of Residential were misclassified as Grass/Crops. The Sparse Grass regions were very small, thus creating a large amount of between class overlap when using the $21 \times 21$ window. The error introduced by the large arrays was even more apparent along the borders of two homogenous land cover regions with high tonal, between-class contrast. This problem was especially acute in the classified ADAR images, where the pixel size was small and most of the land cover regions had a relatively smooth texture. In most circumstances, the spectral data was superior to the texture data.

In some cases, it was found that the optimal size and optimal size/minimum value window programs were able to improved accuracy by characterizing the within-class variability while reducing between-class edge effect errors. In the SPOT data, the optimal size image reduced errors where Sparse Grass was misclassified as Urban/Barren due to edge effect. Also, errors with the edges of Forest being misclassified as Residential in the spectral image were reduced substantially in the optimal size image. The overall KIAs for the SIR-C data were vastly improved by using the optimal size and optimal size/minimum value texture. These programs were less successful in the ADAR data due to the homogeneity of the land cover classes. One notable exception was the Sunlit Forest class, where there was some improvement in the KIAs when compared to the spectral data. 
Several conclusions can be drawn from this research. This study has evaluated different adaptive texture strategies. Also, by testing this method on three different scales and types of data, it allowed some measure as to the robustness of this approach.

The increasing use of RS data has led to a demand for greater automation in the classification process. It is expected that a large amount of new, high resolution optical and radar data will become available in the near future. The accuracy of conventional, per-pixel classifiers may not always be satisfactory due to the H-Resolution problem in the near-optical data and, the calibration and speckle problems in the radar data. Therefore, extracting the maximum amount of spatial information in the form of texture from an image will become increasingly important. This approach could point to a method for segmenting images based on texture. It appears to be a way of moving towards more sophisticated, multi-scale methods of image analysis, which is conceptually more similar to human vision. 


\section{CHAPTER EIGHT}

\section{RECOMMENDATIONS FOR FURTHER STUDY}

Empirical evidence from this study suggests that three types of within-class texture exist. These three types directly correspond to the scale of objects within an image as proposed by Woodcock and Strahler (1987). First, two or more objects in LResolution can comprise a single pixel, resulting in a mixed pixel. This creates problems for classifiers that rely solely on spectral data. These mixed pixels can diffuse class statistics, thus decreasing separability between classes. Also, these mixed pixels can exist in large quantities within a given land cover region. Many of these pixels will be included when training data is selected for that particular class. This can result in errors of commission for that class, and errors of omission for other classes. An example of this occurred in the SPOT image, where a large number of mixed pixels were selected in the training data for the Residential class. This resulted in boundary areas of Forest and Grass/Crops being misclassified as Residential in the spectral image. These boundary areas were composed of mixed pixels where trees were included in pixels with roads or water. However, the texture images, including the optimal size window image, were not subject to this error.

A second type of within-class texture exists when the objects are at or near the same size as the pixels. Under these circumstances, a very coarse texture may exist, resulting from the large range of the widely separated DN values.

Finally, the third type of within-class texture exists when objects are in $\mathrm{H}$ Resolution when compared to the size of the pixels. An example of this scenario was the 
Sunlit Forest class in the ADAR data. Here the ground scene was composed of three different types of objects in H-Resolution: individual trees, their shadows, and bare ground. Each of these individual objects was comprised of dozens of pixels. The 21x21 texture image was best at characterizing this texture, followed by the optimal size and optimal size/minimum value texture images. It is evident that large windows were needed in order to obtain a large enough representative sample of pixels. In this case, larger window arrays may have improved the classification accuracy of this class even further.

Although the optimal size window model seems to have improved the accuracy of the classifications, it may be necessary to incorporate other texture measures into this model to better cope with these three types of within-class texture. The standard deviation of a window is a good measure of the average amount of dispersion of DN values. However in some cases, it was unable to accurately represent two distinct land cover regions. An example of this was the Urban/Barren class in the SPOT data. Both of these land covers had very similar spectral characteristics, and similar texture values. Because of the difficulty of separating these classes, they were combined for this study as one land cover class. However, if a different measure of dispersion such as range was included in the model, these two land covers possibly could have been separated into two distinct classes. While they both had the same standard deviation, the Urban cover may have had a much greater range of DN values due to the presence of some trees and pavement. As with the minimum-value window, some comparison between the SDTP curve and the range would be needed in order to ensure that the model was robust and not scene specific. 
The results of this study indicate that future research for optimal size windows for texture analysis should concentrate on four main areas:

1. Incorporate other measures of dispersion, including the range of local DN values, so that texture for a particular land cover region can be specifically identified.

2. Include larger window sizes to deal with H-Resolution texture in high resolution images.

3. Investigate the possibility of reducing intermediate size windows in order to simplify the model and reduce computation time.

4. Develop better windows that adapt in shape near edges of land cover regions. 


\section{$\underline{\text { REFERENCES }}$}

Agbu, P.A., and E. Nizeyimana, 1991: “Comparisons between Spectral Mapping Units Derived from SPOT Image Texture and Field Soil Map Units”. Photogrammetric Engineering andRemote Sensing. 57 (4): 397-405.

Ahearn, S., 1988: "Combining Laplacian Images of Different Spatial Frequencies (Scales): Implications for Remote-Sensing Image Analysis". IEEE Transactions on Geoscience and Remote Sensing, 26 (6): 826-831.

Barber, D.G. and E.F. LeDrew, 1991: "SAR Sea Ice Discrimination Using Texture Statistics:A Multivariate Approach." Photogrammetric Engineering and Remote Sensing. 57 (4): 385-395.

Briggs, J.M., and M.D. Nellis, 1991: "Seasonal Variation of Heterogeneity in the Tallgrass Prairie:A Quantitative Measure Using Remote Sensing”. Photogrammetric Engineering and RemoteSensing. 57 (4): 407-411.

Brivio, P.A., I. Doria, and E. Zilio, 1993: “Aspects of Spatial Autocorrelation of LANDSAT TMData for Inventory of Waste Disposal Sites in Rural Environments." PhotogrammetricEngineering and Remote Sensing. 59: 13771382.

Chavez, Jr., P. and B. Bauer, 1982: “An Automatic Optimum Kernel-Size Selection Technique for Edge Enhancement”. Remote Sensing of Environment. 12: 2338.

Cohen, W.B., T.A. Spies, and G.A. Bradshaw, 1990: "Semivariograms of Digital Imagery for Analysis of Conifer Canopy Structure." Remote Sensing of Environment. 34: 167-178.

Collins, J.B., C.E. Woodcock, and D.L.B. Jupp, 1995: "Spatial Dependence and Nested Hierarchical Scene Models.” Proceedings of the ASPRS Conference, 3: 535-544. 
Dillworth, M.E., J.L. Whistler, and J.W. Merchant, 1994: "Measuring Landscape Structure Using Geographic and Geometric Windows". Photogrammetric Engineering and Remote Sensing. 60 (10): 1215-1224.

Dutra, L.V. and N.D.A. Mascarenhas, 1984: "Some Experiments with Spatial Feature Extraction Methods in Multispectral Classification". International Journal of Remote Sensing. 5 (2): 303-313.

Ferro, C., 1998: "Scale and Texture in Digital Image Classification". Unpublished M.A. Thesis,West Virginia University, Morgantown, West Virginia.

Franklin, S.E. and G.J. McDermid, 1993: "Empirical Relations between Digital SPOT HRV and CASI Spectral Response and Lodgepole Pine (Pinus Contorta) Forest Stand Parameters". International Journal of Remote Sensing. 14 (12): 2331-2348.

Gong, P., D.J. Marceau, and P.J. Howarth, 1992: "A Comparison of Spatial Feature Extraction Algorithms for Land-Use Classification with SPOT HRV Data". Remote Sensing of Environment, 40: 137-151.

Haralick, R.M. and K.S. Shanmugam, 1974: "Combined Spectral and Spatial Processing ofERTS Imagery Data. Remote Sensing of Environment. 3: 3-13.

Hay, G.J., K.O. Niemann, and D.G. Goodenough, 1997: "Spatial Thresholds, ImageObjects, andUpscaling: A Multiscale Evaluation". Remote Sensing of Environment. 62: 1-19.

Hay, G.J., K.O. Niemann, and G.F. McLean, 1996: “An Object-Specific Image-Texture Analysis of H-Resolution Forest Imagery". Remote Sensing of Environment. 55: $108-122$.

Hodgson, M.E., 1991: "Characteristics of the Window for Neighborhood Analysis of Nominal Data". ASPRS Technical Papers, 1991 ASPRS Annual Convention, 3: 206-214.

Hodgson, M. E., 1998: “What Size Window for Image Classification? A Cognitive 
Perspective". Photogrammetric Engineering and Remote Sensing. 64 (8): 797 807.

Hsu, S., 1978: "Texture-Tone Analysis for Automated Land-Use Mapping". Photogrammetric Engineering and Remote Sensing. 44 (11): 1393-1404.

Jensen, J.R., 1979: "Spectral and Textural Features to Classify Elusive Land Cover at the Urban Fringe". The Professional Geographer, 4: 400-409.

Jensen, J.R., 1996: "Chapter 7, Image Enhancement". In: Introductory Digital Image Processing: A Remote Sensing Perspective, $2^{\text {nd }}$ Edition, Prentice Hall, Upper Saddle River, NJ. 153-165, 186-192.

Johnsson, K., 1994: "Segment-Based Land-Use Classification from SPOT Satellite Data". Photogrammetric Engineering and Remote Sensing. 60 (1): 47-53.

Kramer, H.J., 1996: Observation of the Earth and Its Environment. Survey of Missions and Sensors. Springer Verlag, Berlin, $960 \mathrm{pp}$.

Latty, R.S., R. Nelson, B. Markham, D. Williams, D. Toll, and J. Irons, 1985:

"Performance Comparisons Between Information Extraction Techniques Using Variable Spatial Resolution Data". Photogrammetric Engineering and Remote Sensing. 51 (9): 1459-1470.

Marceau, D.J., P.J. Howarth, J.M. Dubois, and D.J. Gratton, 1990: "Evaluation of the Grey-Level Co-occurrence Matrix Method for Land-Cover Classification Using SPOT Imagery". IEEE Transactions on Geoscience and Remote Sensing. 28 (4): 513-519.

McDermid, G.J. and S.E. Franklin, 1994: "Spectral, Spatial, and Geomorphic Variables for the Remote Sensing of Slope Processes", Remote Sensing of Environment, 49: $57-71$.

Merchant, J.W., 1984: "Using Spatial Logic in Classification of Landsat TM Data". Proceedings of the Pecora IX Symposium, Sioux Falls, South Dakota. pp. 378385. 
Nellis, D.M. and J.M. Briggs, 1989: "The Effect of Spatial Scale on Konza Landscape Classification Using Textural Analysis". Landscape Ecology. 2(2): 93-100.

Palmer, P.L. and M. Petrou, 1997: "Locating Boundaries of Textured Regions". IEEE Transactions on Geoscience and Remote Sensing. 35 (5): 1367-1371.

Richards, 1993: Remote Sensing Digital Image Analysis: An Introduction, Springer Verlag, Berlin, 340pp.

Riou, R. and F. Seyler, 1997: "Texture Analysis of Tropical Rain Forest Infrared Satellite Images". Photogrammetric Engineering and Remote Sensing. 63: 515521.

Ryherd, S. and C. Woodcock, 1996: "Combining Spectral and Texture Data in the Segmentation Remotely Sensed Images". Photogrammetric Engineering and Remote Sensing. 62 (2): 181-194.

Schunck, B., 1987: "Edge Detection with Gaussian Filters at Multiple Scales". Proceedings IEEE Computer Society Workshop on Computer Vision, Miami Beach, Florida. pp. 208-210.

Swain, P.H. and S.M. Davis, 1978. Remote Sensing: The Quantitative Approach. McGraw Hill, New York, 369 pp.

Wang, Li and D.C. He, 1990: "A New Statistical Approach for Texture Analysis". Photogrammetric Engineering and Remote Sensing. 56 (1): 61-66.

Woodcock, C.E. and A.H. Strahler, 1987: "The Factor of Scale in Remote Sensing". Remote Sensing of Environment. 21: 311-332.

Woodcock, C.E., A.H. Strahler, and D.L. Jupp, 1988a: "The Use of Variograms in Remote Sensing. I. Scene Models and Simulated Images." Remote Sensing of Environment, 25: 323-348. 
Woodcock, C.E., A.H. Strahler, and D.L. Jupp, 1988b: "The Use of Variograms in Remote Sensing. II. Scene Models and Simulated Images." Remote Sensing of Environment, 25: 349-379. 